\title{
Synthesis and influence of alkaline concentration on $\alpha$-FeOOH nanorods shapes
}

\author{
ZHANG ENLEI*, WANG GUOSHENG, LONG XIAOZHU and WANG ZHUMIN \\ College of Chemical Engineering, Shenyang University of Chemical Technology, Shenyang 110142 , \\ People's Republic of China
}

MS received 13 March 2013; revised 3 June 2013

\begin{abstract}
FeOOH nanorods having uniform morphology were synthesized by water bath process. X-ray diffraction and transmission electron microscopy revealed that the single-crystalline orthorhombic $\alpha$-FeOOH nanorods are about $10 \mathrm{~nm}$ in diameter and hundreds of nanometer in length. The effect of concentration of KOH on morphology of $\alpha$-FeOOH nanorods was investigated. pH values of solution play an important role in the process of transformation of ferrihydrite into goethite. The inconsistent growth rate along different facets can be effected by $\mathrm{pH}$ values of the solution. The influence and growth mechanism of $\alpha$-FeOOH nanorods were discussed in detail. This method may be widely used as reference to fabricate other inorganic onedimensional nanostructured materials and easily realized in industrial-scale synthesis.
\end{abstract}

Keywords. $\alpha$-FeOOH; nanorods; shape control.

\section{Introduction}

The physical and chemical properties of nanoparticles are essentially different from those of bulk materials and largely dependent on their size, shape and surface chemistry (Tang et al 2006; Cwiertny et al 2009). Iron oxides have attracted much attention because of many interesting properties that make them to be used in a wide range of applications such as catalysts (Zheng et al 2006), electrochemical devices (Wang et al 2004), magnetic storages (Lemaire et al 2002), sensors (Chen et al 2005) and mineral liquid crystals (MLCs) (Lemaire et al 2002), etc.

Goethite $(\alpha-\mathrm{FeOOH})$, as one of the most important iron compounds, is of considerable importance in several technological applications such as catalysts, gas sensors, magnetic recording media and raw materials. Goethite shows a strong affinity for a variety of contaminants such as heavy-metal ions and organic pollutants and can be used as a starting material in the production of red pigment. Because of its unique properties, a considerable amount of successful method has been developed to prepare goethite. For meeting the ever increasing technological demand, it is still a challenge to modify size of nanostructured goethite. In order to obtain goethite nanocrystals, various synthetic procedures have been introduced in the past decade. These approaches included thermal decomposition (Fu et al 2003), hydrothermal approach (Giri et al 2005), sol-gel process (Woo et al 2003) and others (Jin et al 2004; Varanda et al 2005). However, these methods

*Author for correspondence (zhangel1979@126.com) often were used as hard templates or surfactant. Thus, high yield synthesis of pure goethite nanorods with high aspect ratio still remains a challenge to be considered for above-mentioned disadvantage. In this work, we have developed a simple method for synthesizing pure goethite nanorods by water bath process without any hard template or surfactant. The synthesized goethite nanorods were about $10 \mathrm{~nm}$ in diameter. The crystal growth direction $\langle 100\rangle$ is clearly observed. It was found that morphology of the products changes depending upon the intial $\mathrm{KOH}$ concentration.

\section{Experimental}

All the chemicals were of analytical grade and used without further purification. $\alpha$-FeOOH nanorods were synthesized according to following procedure: $20 \mathrm{~mL}$ of $\mathrm{KOH}$ $(5-18 \mathrm{M})$ was added in a mixed solution of $80 \mathrm{~mL}$ $\mathrm{FeCl}_{3} \cdot 6 \mathrm{H}_{2} \mathrm{O}(0 \cdot 375 \mathrm{M})$. The mixture was stirred for $3 \mathrm{~h}$ until an orange solution was obtained. The solution was heated at $80{ }^{\circ} \mathrm{C}$ at a rate of $2{ }^{\circ} \mathrm{C} / \mathrm{min}$ and then maintained for $4 \mathrm{~h}$. After reaction, the solution was naturally cooled to room temperature. The brown precipitation was collected by centrifugation and washed with deionized water and ethanol for some time. The products were finally dried in a free-drying apparatus for $24 \mathrm{~h}$. To investigate the role of $\mathrm{KOH}$, in addition, a $\mathrm{KOH}$-free control experiment was further carried out under the same reaction conditions.

The phase of the products was characterized by X-ray powder diffraction (XRD, Panalytical Xpert PRO 
diffractometer with $\mathrm{CuK} \alpha$ radiation). The morphologies and internal structures of the samples were characterized by high-resolution transmission electron microscopy (HRTEM, TECNAI F-30 with an accelerating voltage of $300 \mathrm{kV})$.

\section{Results and discussion}

The uniform morphology of $\alpha$-FeOOH nanorods were prepared by water bath process without using any hard template or surfactant. The crystal phase and structure information of the nanorods were obtained by XRD measurement. XRD pattern for obtained sample is shown in figure 1. As can be seen from the pattern, the major diffraction peaks can be indexed as the reflections of pure orthorhombic $\alpha$-FeOOH with lattice constants of $a=$ $4.608 \AA, b=9.956 \AA$ and $c=3.0215 \AA$. These values are almost identical to known values for $\alpha$-FeOOH (JCPDS card no. 29-0713). The strong and sharp diffraction peaks indicate that the highly crystalline nature of the $\alpha$-FeOOH nanorods. No other impurities have been found in the synthesized products.

The morphology of the as-obtained products were examined by the field emission scanning electron microscopy (FESEM), whereby the solid sample was mounted on a copper slice after ultrasonic dispersion treatment. FESEM image in figure 2 shows that the products consist almost entirely of such $\alpha$-FeOOH one-dimensional nanostructures and this indicates the high yield and good uniformity achieved with this approach.

A typical TEM image of the sample synthesized by water bath process is shown in figure 3(a), whereby the sample was treated with ultrasonic dispersion in ethanol for $30 \mathrm{~min}$ and put onto a copper grid. Its corresponding local enlargement (figure $3 \mathrm{~b}$ ) reveals that the nanorods are of about $10 \mathrm{~nm}$ in width and hundreads of nanometer in length, and the synthesized $\alpha$-FeOOH nanorods were

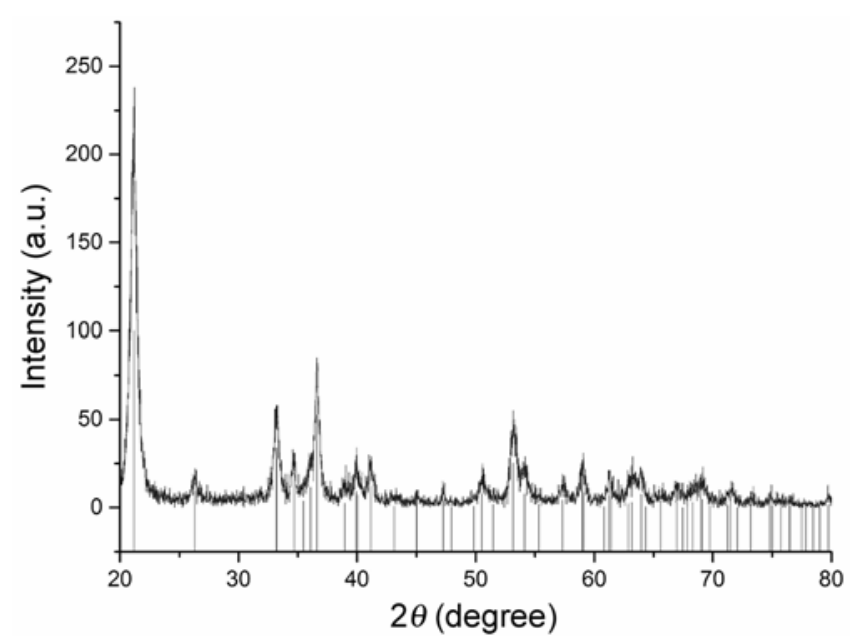

Figure 1. XRD pattern of $\mathrm{FeOOH}$ nanorods. very clean and straight. More detailed structural information on the as-synthesized self-assembled nanorods was further provided by HRTEM. Figure 4 shows a representative HRTEM image of an individual nanorod, clearly revealing that the as-obtained nanorods are structurally uniform and crystalline. The interdistance of lattice planes is $\sim 0.418 \mathrm{~nm}$, which can be indexed to the (110) plane, further confirming that the nanorods are crystalline and grow along [001] axis.

In water, the particle surfaces are hydrated, thus, the effect of alkaline concentration is one of the dominant factors in the hydrolysis reaction which can affect the saturation of ions in solution, and hence the nucleation and growth of particles. In this study, effect of $\mathrm{KOH}$ solution concentration was tested at $80{ }^{\circ} \mathrm{C}$ in the range of 5-18 $\mathrm{M}$ and the corresponding particles with different morphologies and sizes were shown in figure 5. It was found that aspect ratio of the nanorods changes depending upon the intial $\mathrm{KOH}$ concentration: higher concentrations would yield smaller and narrower nanorods under the reported conditions and the shape would turn nanorods into nanoparticles. For example, nanorods show a diameter of about $30 \mathrm{~nm}$ and lengths upto hundred nanometer when the concentration of $\mathrm{KOH}$ was fixed at $5 \mathrm{M}$ (figure 5a); while the diameter of nanorods decreases to $10 \mathrm{~nm}$ as the concentration of $\mathrm{KOH}$ was fixed at $12 \mathrm{M}$ (figure 5b); while the diameter of the nanorods decrease to $4 \mathrm{~nm}$ (figure $5 \mathrm{c}$ ). In addition, the size control of uniform nanorods was mainly limited in the concentration range of 5-12 $\mathrm{M}$ under our reported conditions. A further increase in the concentration could cause a wide size distributon of particle including the presence of spherical particles, as shown in figure 5(c). When the concentration of $\mathrm{KOH}$ was fixed at $15 \mathrm{M}$, the product consists of

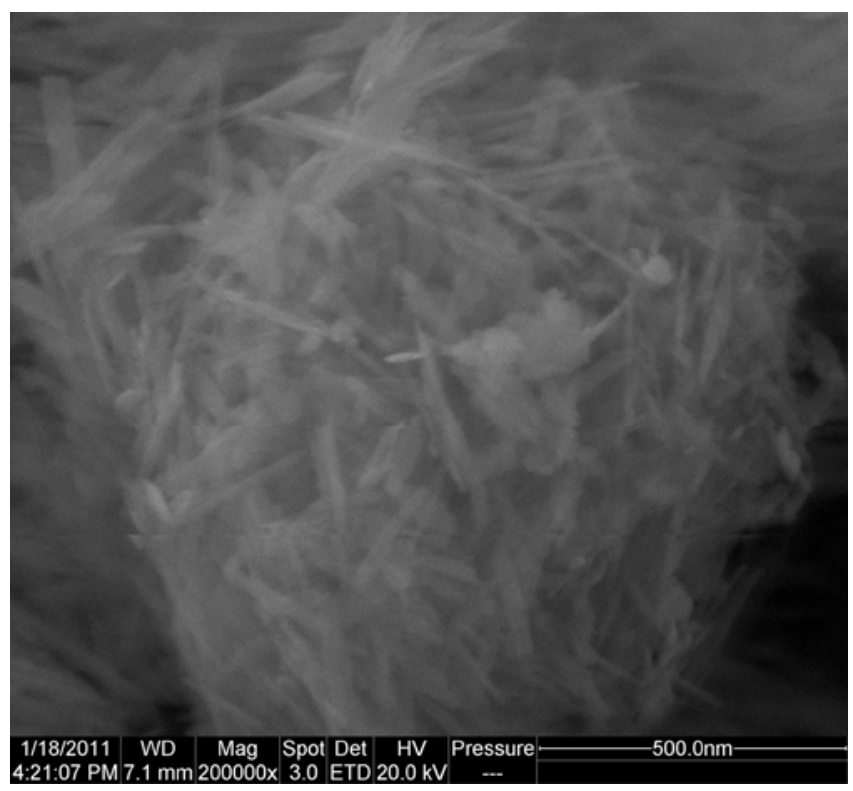

Figure 2. SEM image of $\alpha-\mathrm{FeOOH}$ nanorods. 

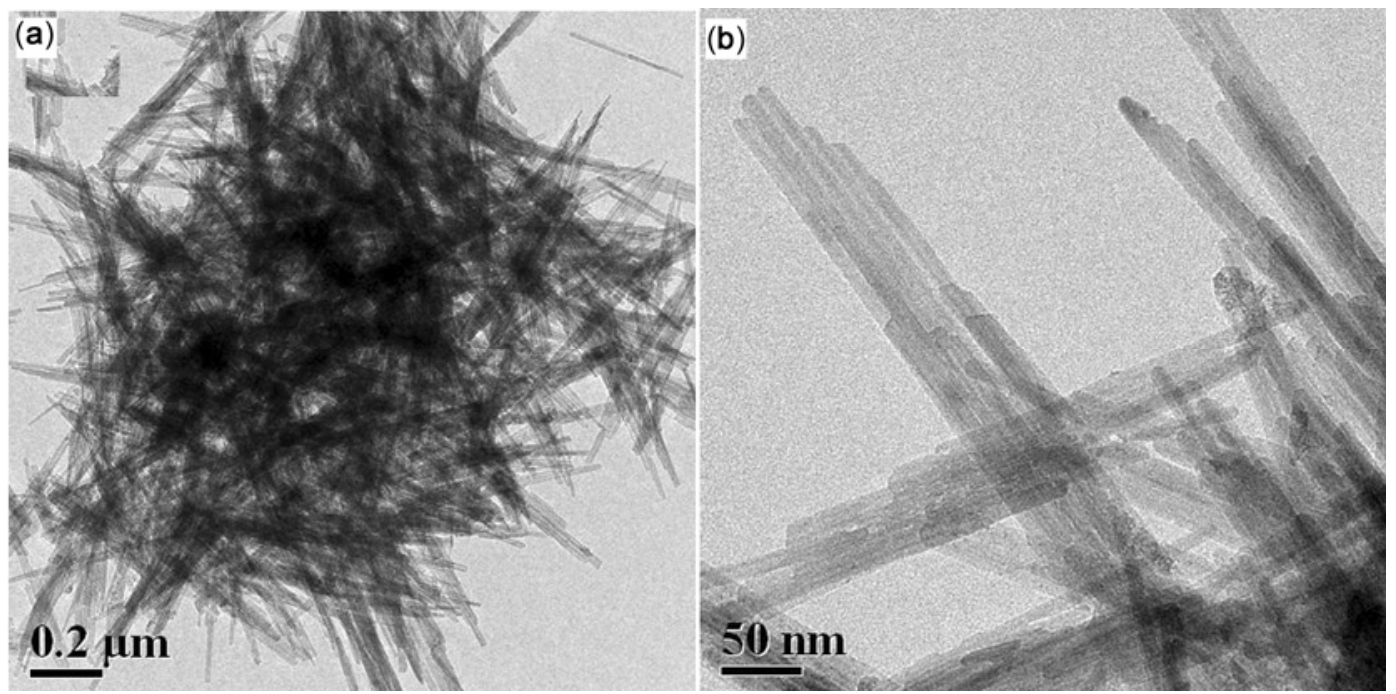

Figure 3. TEM image of $\alpha-\mathrm{FeOOH}$ nanorods (a) low magnification and (b) high magnification.

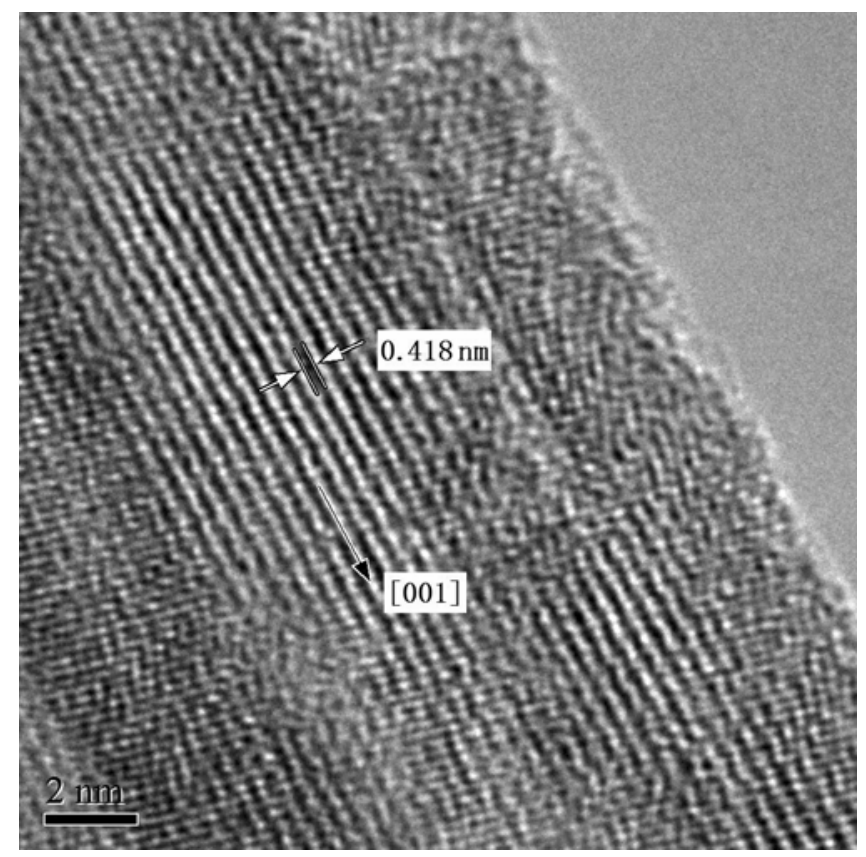

Figure 4. HRTEM image of $\alpha$-FeOOH nanorods.

nanoparticles with a size of about $3 \mathrm{~nm}$, as shown in figure $5(\mathrm{~d})$. The crystal structure of these particles was characterized and analysed using XRD technique, as shown in figure 6 . The pattern of nanorods prepared with different concentrations of $\mathrm{KOH}$ shows that all the diffraction peaks could be assigned to the pure orthorhombic $\alpha$-FeOOH with lattice constants of $a=4.608 \AA$, $b=9.956 \AA$ and $c=3.0215 \AA$, consistent with the reported values (JCPDS no. 29-0713).

In the case of oxidation of iron, the first phase to precipitate hydrolysis reaction of $\mathrm{Fe}^{3+}$ ions is usually ferrihydrite, a poorly crystalline oxide, of rough formula $5 \mathrm{Fe}_{2} \mathrm{O}_{3} \cdot 9 \mathrm{H}_{2} \mathrm{O}$ (Loan et al 2005). Nevertheless, the exact mechanisms of formation of goethite from the precursor ferrihydrite are not clearly established. Schwertmann et al (2004) revealed that the $\mathrm{pH}$ values of solution play an important role in the process of transformation of ferrihydrite into goethite. According to Schwertmann's study, in alkaline solutions, the dissolution of ferrihydrite follows the equilibrium (1)

$$
\begin{aligned}
& 5 \mathrm{Fe}_{2} \mathrm{O}_{3} \cdot 9 \mathrm{H}_{2} \mathrm{O}_{(\mathrm{S})}+(10 n-30) \mathrm{OH}_{(\mathrm{aq})}^{-}+(66-10 n) \mathrm{H}_{2} \mathrm{O} \rightarrow \\
& 10\left[\mathrm{Fe}(\mathrm{OH})_{n}\left(\mathrm{H}_{2} \mathrm{O}\right)_{(6-n)}\right]_{(\mathrm{aq})}^{(n-3)-}(3<n \leq 6) .
\end{aligned}
$$

In our experiments, we think that the value of $n$ is 6 at very alkaline $\mathrm{pH}$ and ferrihydrite is dissolved under the 

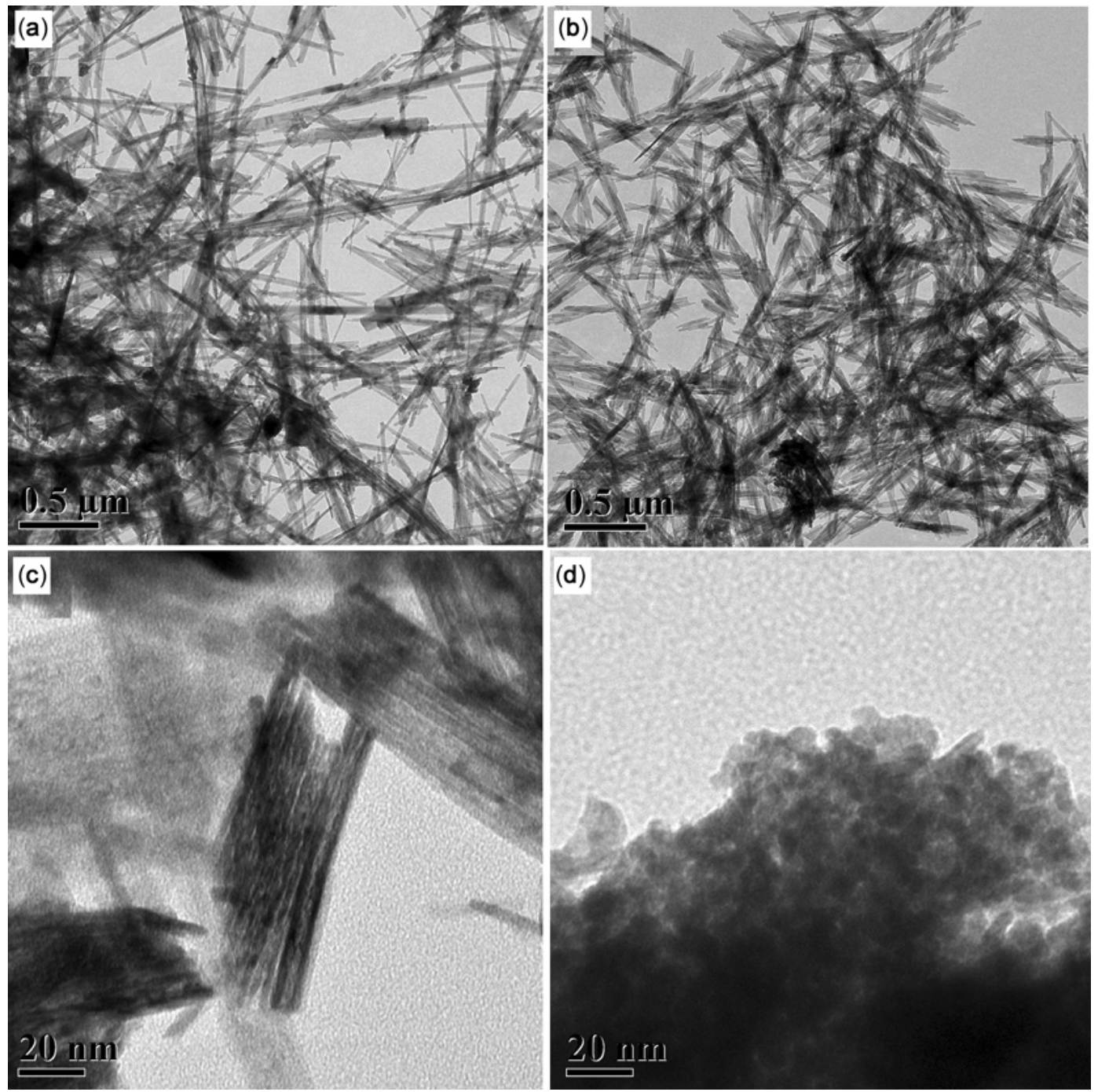

Figure 5. TEM image of products synthesized under adding different concentrations of $\mathrm{KOH}$ solution: (a) $5 \mathrm{M}$; (b) $12 \mathrm{M}$; (c) $15 \mathrm{M}$ and (d) $18 \mathrm{M}$.

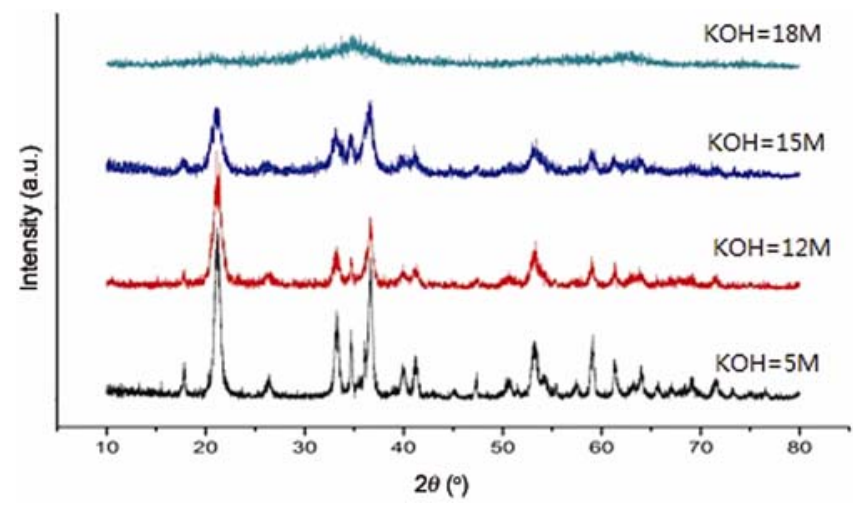

Figure 6. XRD pattern of products synthesized under adding different concentrations of $\mathrm{KOH}$ solution.

complex anions, $\mathrm{Fe}(\mathrm{OH})_{6}^{3-}(n=6)$ eventually. So at appropriate temperature, the precursor, $\mathrm{Fe}(\mathrm{OH})_{6}^{3-}$ as condensed by olation and oxolation processes between $\mathrm{OH}$ and $\mathrm{H}_{2} \mathrm{O}$ ligands, which give rise to $\mathrm{Fe}-\mathrm{OH}-\mathrm{Fe}$ and $\mathrm{Fe}-\mathrm{O}-\mathrm{Fe}$ bridges, formed the nucleation of goethite. And then, other precursor, $\mathrm{Fe}(\mathrm{OH})_{6}^{3-}$ as aggregated on the nucleation of goethite, lead to the growth of goethite. The fringes of an individual nanowire are not intact, which were observed in figure 4(b and c), can be explained by the precursor, $\mathrm{Fe}(\mathrm{OH})_{6}^{3-}$ aggregated from in to out in the growth process of goethite. The inconsistent growth rate along different facets can be explained by the 'lowest energy' argument resulting in needlike crystals. The nanowires morphology is thermodynamically favoured as it allows extension of the higher energy surfaces with respect to lower ones (Michael et al 2003). This is advantageous for the growth of $\alpha-\mathrm{FeOOH}$ nanowires along [001] axis.

\section{Conclusions}

In conclusion, we report large-yield synthesis of $\alpha-\mathrm{FeOOH}$ nanorods with high aspect ratio using $\mathrm{FeCl}_{3} \cdot 6 \mathrm{H}_{2} \mathrm{O}$ and $\mathrm{KOH}$ as starting reactants by water bath process at $80{ }^{\circ} \mathrm{C}$ 
for $4 \mathrm{~h}$. Structural characterization by XRD revealed that the $\alpha$-FeOOH product has an orthorhombic structure. Morphological examination by FESEM and TEM revealed that the orthorhombic of $\alpha$-FeOOH product has a nanorod structure with a diameter of $10 \mathrm{~nm}$ and length up to hundreds of nanometer. HRTEM showed that the product is of single crystallinity. The aspect ratio of the nanorods changes depending upon the initial $\mathrm{KOH}$ concentration. The present method is simple, mild, low-cost without using hard template or surfactant and large-production, which will be used to synthesize homogeneous nanostructures of other nanowire oxides at relatively low temperature and realize industrial-scale synthesis.

\section{References}

Chen J, Xu L N, Li W Y and Gou X L 2005 Adv. Mater. 17582 Cwiertny D M, Hunter G J, Pettibone J M, Scherer M M and Grassian V H 2009 J. Phys. Chem. C113 2175

Fu Y Y, Wang R M, Xu J, Chen J, Yan Y, Narlikar A V and Zhang H 2003 Chem. Phys. Lett. 379373
Giri S, Samanta S, Maji S, Ganguli S and Bhaumik A J 2005 Magn. Magn. Mater. 285296

Jin J, Ohkoshi S I and Hashimoto K 2004 Adv. Mater. 16 48

Lemaire B J, Davidson P, Ferre J, Jamet J P, Anine P P, Dozov I and Jolivet J P 2002 Phys. Rev. Lett. 88125507

Loan M, Parkinson G M and Richmond W R 2005 Am. Miner. 90258

Michael B S J, Ali G, Tobias H, Aaron E S, Frank L and Brian A K 2003 J. Am. Chem. Soc. 12516050

Schwertmann U, Stanjek H and Becher H H 2004 Clay Miner. 39433

Tang B, Wang G L, Zhou L H, Ge J C and Cui L J 2006 Inorg. Chem. 455196

Varanda L C, Morales M P, Jafelicci M and Serna C J 2002 J. Mater. Chem. 123649

Wang X, Chen X Y, Gao L S, Zheng H G, Ji M R, Tang C M, Shen T and Zhang Z D 2004 J. Mater. Chem. 14905

Woo K, Lee H J, Ahn J P and Park Y S 2003 Adv. Mater. 15 1761

Zheng Y H, Chen Y, Wang Y S, Bao F, Zhou L H, Wei X F, Zhang Y Y and Zheng Q 2006 J. Phys. Chem. B110 3093 